\author{
Original
}

\title{
Investigation of hand impact on PIFA performances and SAR in human head
}

\author{
Md. Ikbal Hossain ${ }^{\mathrm{a}, *}$, M.R. Iqbal Faruque ${ }^{\mathrm{a}}$, M. Tariqul Islam ${ }^{\mathrm{b}}$ \\ ${ }^{a}$ Space Science Centre (ANGKASA), Research Centre Building, Universiti Kebangsaan Malaysia (UKM), Bangi, Selangor, Malaysia \\ ${ }^{\mathrm{b}}$ Department of Electrical, Electronic and Systems Engineering, Faculty of Engineering \& Built Environment Building, \\ Universiti Kebangsaan Malaysia (UKM), Bangi, Selangor, Malaysia
}

Received 9 October 2014; accepted 27 May 2015

\begin{abstract}
The aim of this investigation is to analyze the effects of hand on planar inverted-F antenna (PIFA) electromagnetic (EM) absorption and performances. The EM absorption is evaluated by using two parameters: the specific absorption rate (SAR) in the human head and total absorbed power by the user. The antenna performance parameters comprising return loss, radiation efficiency, and gain are considered in this investigation. The analysis was performed using mobile phone with a human head and hand model in both cheek and tilt positions of talk mode. The results show that the inclusion of hand model leads to decrease the SAR and increase the total absorbed power by user remarkably. The hand phantom leads maximum $27.2 \%$ SAR reduction in both cheek and tilt positions of talk mode. Moreover, the hand phantom contributed to increase the total power absorption more than two times in both cheek and tilt positions. The user's hand also leads to degrade antenna performances considerably. Moreover, the presented analysis provides some useful indication to design handset antenna considering hand effects.

All Rights Reserved (C) 2015 Universidad Nacional Autónoma de México, Centro de Ciencias Aplicadas y Desarrollo Tecnológico. This is an open access item distributed under the Creative Commons CC License BY-NC-ND 4.0.
\end{abstract}

Keywords: Antenna; Electromagnetic (EM) radiation; Human hand model; Human head model; Specific Absorption Rate (SAR)

\section{Introduction}

The portable terminal devices like cell phone are being popular day by day and hence the number of cell phone users is increasing dramatically. The antennas of the cellular devices receive and radiate electromagnetic (EM) energy of different frequency depending on the type of applications. The emitted EM energy from portable terminal device's antenna may cause serious health hazards (Repacholi, 2001). Because, the EM energy can penetrate human tissues and a part of the energy is absorbed in the body. The power absorption by human body tissue is usually evaluated in terms of specific absorption rate (SAR) (Hossain et al., 2015). The safety limits of the SAR values for portable devices are imposed by the Federal Communication Commission (FCC) in USA and International Commission on Non-Ionizing Radiation protection (ICNIRP) in Europe. The SAR values can be affected by varying antenna geometry, radiated power, frequency of exposure, spacing between antenna and human head, and exposure medium (Faruque et al., 2014). The easiest way to reduce the SAR is to

\footnotetext{
*Corresponding author.

E-mail address: ipk_eee@yahoo.com (Md. Ikbal Hossain).
}

increase the spacing between the antenna and human body (Kivekas et al., 2004). On the other hand, a number of EM absorption reduction techniques such as conductive shielding, ferrite shielding, electromagnetic band gap (EBG) surface, and metamaterial techniques can be applied to reduce the EM radiation effects on human health (Hossain et al., 2014).

The human body acts as a lossy dielectric medium. The relative permittivity of biological tissues are high compared to the permittivity of air (Valkonen et al., 2011). The relatively high permittivity of the human hand (holding mobile phone) can change of impedance in the near field zone of the antenna. In addition, the human body tissues have also a high water content, which makes them moderately conductive. The conductive properties of human hand causes losses at microwave frequencies. So, the inclusion of the hand model definitely affects antenna performances (Ilvonen et al., 2011). In Li et al. (2009), an investigation of effects of hand phantom on planar inverted-F antenna (PIFA) and helical antenna was presented. The results indicated that hand phantom leads to affect antenna performances depending on the position of hand with respect to mobile phone antenna. The user's hand can cause shifting the resonance frequency, reducing the channel data rate and detuning the antenna input impedance (Zhao et al., 2013). In Ve- 
hovsky et al. (2014), an analysis of hand effects on the resonance characteristics of dual band PIFA was presented. The results showed that the hand phantom brought slight impedance matching deterioration for both GSM frequency bands. Additionally, the presence of the user's hand lead to less conservative SAR values. The human hand reduces the SAR values to the head tissues, as it dissipates a part of radiated power of antenna (Okoniewski \& Stuchly, 1996). The effects of hand holding position on the EM interaction between mobile phone and human was investigated in Al-Mously and Abousetta (2009). The obtained results indicated that the SAR values remarkably change in consequence of the positioning of the mobile phone against the human head at different hand levels.

The objective of this analysis is to investigate the effects of the human hand on PIFA EM absorption characteristics and performances for two different GSM frequency bands. Two parameters (SAR and total absorbed power) are used to explain the EM absorption characteristic. The commonly used cheek and tilt position of talk mode are used to simulate the handset antenna with the user's head and hand phantoms. Moreover, the effects on the antenna performance parameters comprising radiation efficiency and gain were investigated to evaluate the effects of hand phantom on antenna performances.

\section{Method and Materials}

A dual band GSM (0.9 GHz and $1.8 \mathrm{GHz})$ PIFA is designed for the investigation. The antenna with circuit board, LCD dis-

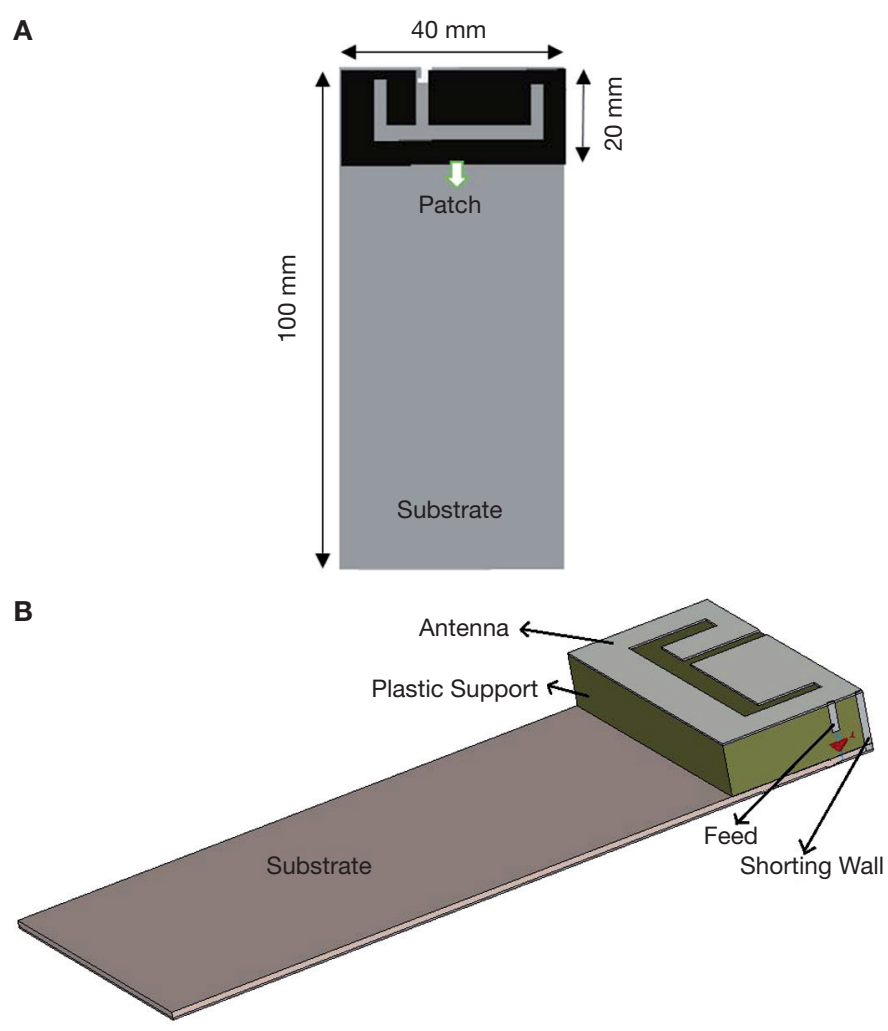

Fig. 1. Geometry of PIFA antenna. A: front view. B: 3D view. play and plastic mobile cover was used in this investigation. The antenna consists of patch, ground, feed and shorting point. The dimensions of patch are $40 \times 20 \times 0.3 \mathrm{~mm}$, which is made with copper sheet. The distance between the substrate and patch is $8 \mathrm{~mm}$, and a plastic support is placed between them. The distance between feeding and shorting plane is $6 \mathrm{~mm}$, and the dimensions of shorting plane are $8 \times 1 \times 0.3 \mathrm{~mm}$. Figures $1 \mathrm{~A}$ and $\mathrm{B}$ indicate the front view and $3 \mathrm{D}$ view of proposed PIFA with FR-4 substrate respectively.

The electrical properties of handset elements were taken to comply with industry standards as indicated in Table 1 . The three-dimensional anatomical models were used for head and hand phantom. The head model provided by the whole brain atlas website is used in this investigation. The head phantom consists of various types of tissues: bone, brain, skin, muscle, fat, and eye. The electrical properties of head and hand model are listed in Table 2 (Quispe-Siccha et al., 2012). The head and hand dielectric properties were set in accordance with the frequency of exposure. The numerical simulation of SAR values and other antenna performance parameters were evaluated adopting the finite-difference time-domain (FDTD) method of the Computer Simulation Technology (CST) Microwave Studio. The Figure 2 shows the simulation setup indicating the relative positions of the mobile handset, human head and hand phantom. For the calculation of SAR values, following standard formula was used (Faruque et al., 2014):

$$
S A R=\frac{\sigma|E|^{2}}{2 \rho}
$$

where, $\sigma$ represents the conductivity of head tissue, E denotes the root mean square (rms) of induced electric field strength, and $\rho$ is tissue density.

The SAR values averaged over $1 \mathrm{~g}$ biological tissue were evaluated adopting IEEE standard algorithm. The stimulated power $0.5 \mathrm{~W}$ was used in experimental set-up and the SAR calculation was performed in the post processing phase of the simulation. In lossy-Drude simulation model, $128 \times 128 \times 128$ cells domain was used with cell size were $\Delta x=\Delta y=\Delta z=3 \mathrm{~mm}$.

Table 1

Electrical properties of handset.

\begin{tabular}{lll}
\hline Handset Materials & Conductivity (S/m) & Relative Permittivity \\
\hline Circuit Board (FR-4) & 0.05 & 4.9 \\
LCD Display & 0.02 & 3 \\
Mobile Cover & 0.027 & 3.5 \\
\hline
\end{tabular}

Table 2

Electrical properties of human head and hand phantoms.

\begin{tabular}{lllll}
\hline Material & \multicolumn{2}{c}{ Conductivity $(\mathrm{S} / \mathrm{m})$} & \multicolumn{2}{c}{ Relative Permittivity } \\
\cline { 2 - 5 } & $0.9 \mathrm{GHz}$ & $1.8 \mathrm{GHz}$ & $0.9 \mathrm{GHz}$ & $1.8 \mathrm{GHz}$ \\
\hline Muscle & 1.50 & 1.35 & 55.95 & 54.44 \\
Skin & 1.48 & 1.33 & 49.60 & 46.90 \\
Brain & 1.11 & 1.09 & 41.7 & 39.50 \\
Bone & 0.12 & 0.11 & 4.83 & 4.48 \\
Fat & 0.13 & 0.12 & 5.10 & 4.91 \\
Eye & 2.03 & 1.99 & 68.6 & 65.3 \\
Hand & 1 & 0.97 & 20 & 18.8 \\
\hline
\end{tabular}


A

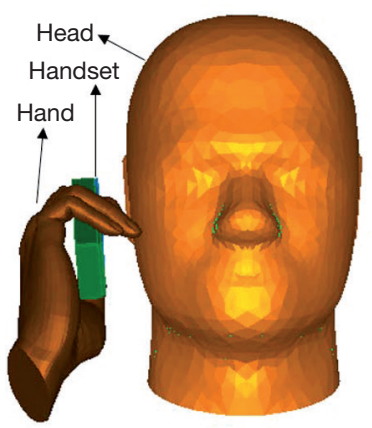

B

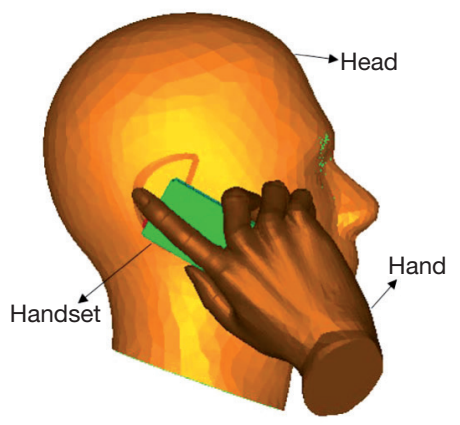

Fig. 2. Simulation setup $15^{\circ}$ tilt position. A: front view. B: side view.

The mesh density control of line per wavelength of 8 , mesh line ratio limit of 200. A total of 6,211,119 mesh cells was generated, and the simulation time was $8208 \mathrm{~s}$ for each run on an Intel Core $^{\mathrm{TM}}$ i5 $34703.20 \mathrm{GHz}$ CPU with 4 GB RAM.

\section{Results and Discussion}

\subsection{Effects of Hand on SAR}

The effects of the human hand on SAR values were studied for cheek and tilt position of talk mode. Only $1 \mathrm{~g}$ SAR value is presented in this paper for all cheek and tilt position analysis. The total absorbed power by head was also studied in every case so that the effect of hand could be separated clearly. The results of effects of hand on the SAR are presented in two subtitles: cheek position analysis and tilt position analysis.

\subsubsection{Cheek Position Analysis}

In cheek position analysis, two different distances $(16 \mathrm{~mm}$ and $18 \mathrm{~mm}$ ) of mobile phone from the head axis were considered both for without the hand and with hand simulation setup. Table 3 represents the SAR results with 3D SAR distribution in head and Figure 3 represents the total absorbed power values by the user for different cheek positions. The results exhibit that the SAR values are reduced significantly due to the presence of a user's hand in all cases comparing with without hand configuration. In a cheek position of the cell phone with $16 \mathrm{~mm}$ apart from the user's head axis, the SAR reduction occurs $27.2 \%$ at the lower GSM frequency band and $2.7 \%$ at the upper GSM frequency band. The results also show that the SAR values for the upper frequency band are less affected than that of the lower frequency band. Moreover, cheek position of $18 \mathrm{~mm}$ contributes to reduce the SAR $27.6 \%$ at $0.9 \mathrm{GHz}$ and $6.21 \%$ at $1.8 \mathrm{GHz}$. Increase in phone distance from the user's head reduces the SAR values in every case. It is also vital to mention that the percentage of SAR reduction due to user's hand is not affected greatly for lower frequency band for two different configurations. But the SAR reduction due to user's hand increases with increasing phone distance at the upper frequency band.

The standard SAR limit for portable devices are $1.6 \mathrm{~W} / \mathrm{kg}$ averaged over $1 \mathrm{~g}$ tissue mass and $2 \mathrm{~W} / \mathrm{kg}$ in a $10 \mathrm{~g}$ averaging

mass (Commission and others, 2001). The $1 \mathrm{~g} \mathrm{SAR}$ standard is specified by the FCC and $10 \mathrm{~g}$ SAR limit is set by the ICNRIP. The results indicate that the calculated $1 \mathrm{~g}$ SAR values of all configurations are below the standard SAR limit.

The total power absorbed by the user increases after using the hand phantom with the head model. In case of cheek position

Table 3

SAR values for different cheek positions.

Cheek

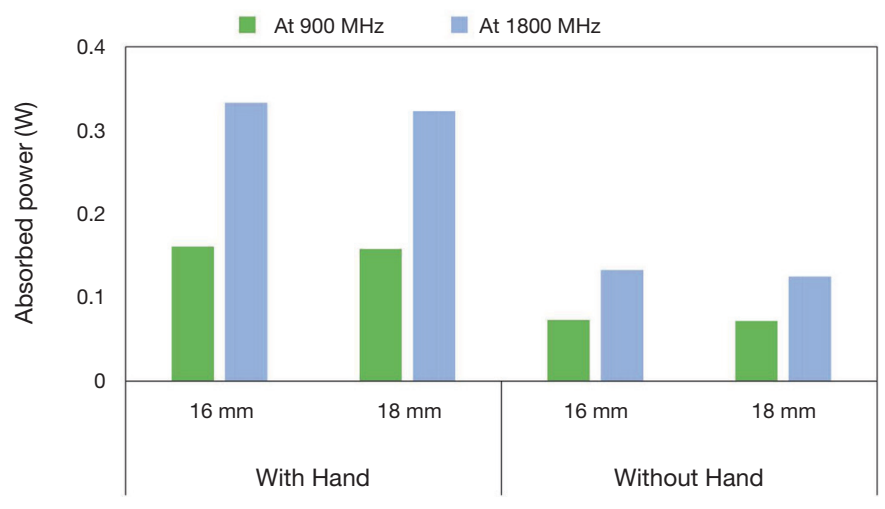

Fig. 3. Total absorbed power by head for different cheek position. 
with $16 \mathrm{~mm}$ phone distance, power absorbed by the user was $32.2 \%$ for with hand configuration and $14.6 \%$ for without hand configuration of the lower frequency band. On the other hand, the power absorbed by the user was $66.6 \%$ and $26.6 \%$ of total radiated power for with and without hand configuration of the upper frequency band considering $16 \mathrm{~mm}$ cell phone distance.

The results from $18 \mathrm{~mm}$ cell phone distance also provide similar tendency as compared with the results from a $16 \mathrm{~mm}$ distance configuration. The user's hand leads to absorbed power more than two times than that of without hand configuration in all cases. However, the inclusion of user's hand leads to reduce the SAR due to the fact that the hand absorb a significant amount of radiated power.

\subsubsection{Tilt Position Analysis}

In this section, $15^{\circ}$ and $30^{\circ}$ tilt position of the cell phone were investigated considering with hand and without hand configuration. Two distance configurations (16 $\mathrm{mm}$ and $18 \mathrm{~mm}$ ) were also used in the tilt position analysis. The results related to the SAR values have been presented in Tables 4 and 5. In case of different tilt position analysis, the SAR values decrease due the presence of the user's hand at $0.9 \mathrm{GHz}$, but the SAR values

Table 4

SAR values for different tilt positions $(16 \mathrm{~mm})$.

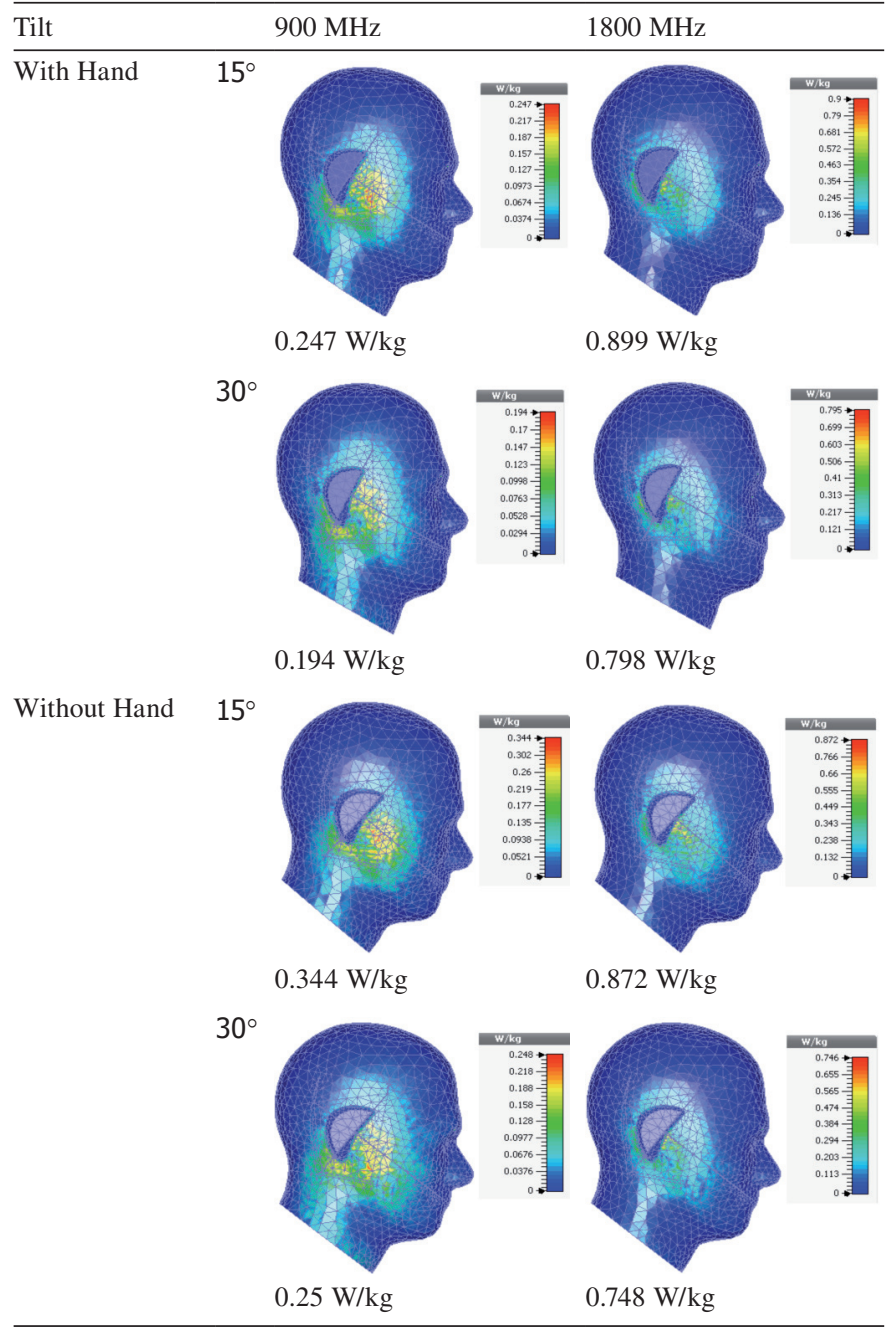

increase at $1.8 \mathrm{GHz}$. For $15^{\circ}$ tilt position of $16 \mathrm{~mm}$ distance, $27.2 \%$ SAR reduction can be obtained at the lower frequency band. On the other hand, the SAR increases $5.14 \%$ at the upper frequency band. In addition, $30^{\circ}$ tilt position of $16 \mathrm{~mm}$ distance configuration contributes to decrease the SAR $26.5 \%$ at $0.9 \mathrm{GHz}$ and increase the SAR $5.8 \%$ at $1.8 \mathrm{GHz}$.

Sequentially, $28.2 \%$ reduction and $3 \%$ increment in the SAR are marked at $0.9 \mathrm{GHz}$ and $1.8 \mathrm{GHz}$ respectively, for $15^{\circ}$ tilt position of $18 \mathrm{~mm}$ distance configuration. $30^{\circ}$ tilt position of $18 \mathrm{~mm}$ distance configuration contributes to decrease the SAR $22.4 \%$ at $0.9 \mathrm{GHz}$ and increase the SAR 5.8\% at $1.8 \mathrm{GHz}$. The results from tilt positions of $20 \mathrm{~mm}$ distance configuration show identical tendencies as two other configurations. The results indicate that the SAR values decrease significantly with increasing tilt angle. Moreover, $8.5 \%$ reduction in the SAR is obtained from $16 \mathrm{~mm}$ to $18 \mathrm{~mm}$ distance configuration in case of $15^{\circ}$ tilt position at $0.9 \mathrm{GHz}$. The SAR decreases $14.4 \%$ from $16 \mathrm{~mm}$ to $18 \mathrm{~mm}$ in case of $15^{\circ}$ tilt position at $1.8 \mathrm{GHz}$ respectively.

The results of absorbed power by the user have been presented in Figure 4 for $16 \mathrm{~mm}$, and in Figure 5 for $18 \mathrm{~mm}$. In tilt position analysis, hand phantom leads to increase total absorbed power more than two times than that of without hand configura-

Table 5

SAR values for different tilt positions $(18 \mathrm{~mm})$.

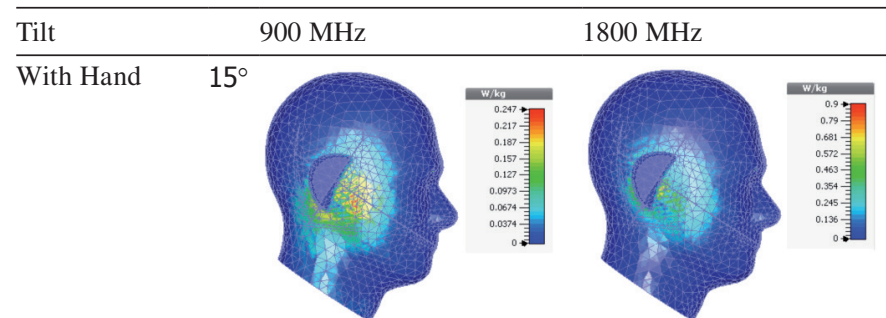

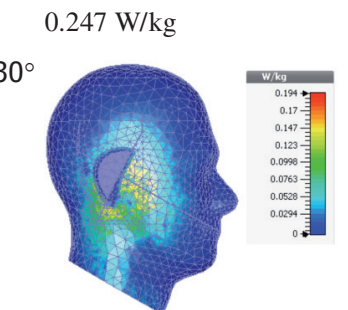

$0.194 \mathrm{~W} / \mathrm{kg}$

Without Hand
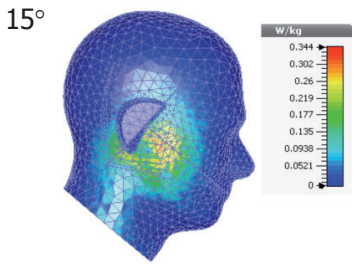

$0.344 \mathrm{~W} / \mathrm{kg}$

$30^{\circ}$

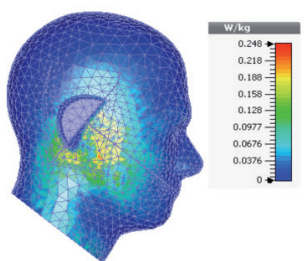

$0.25 \mathrm{~W} / \mathrm{kg}$
$0.899 \mathrm{~W} / \mathrm{kg}$

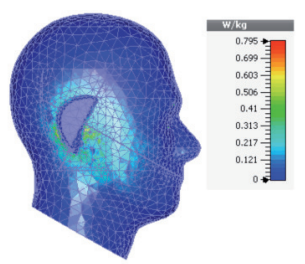

$0.798 \mathrm{~W} / \mathrm{kg}$

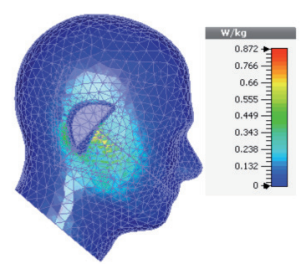

$0.872 \mathrm{~W} / \mathrm{kg}$

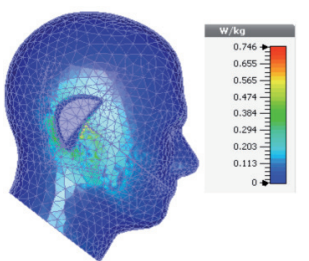

$0.748 \mathrm{~W} / \mathrm{kg}$ 


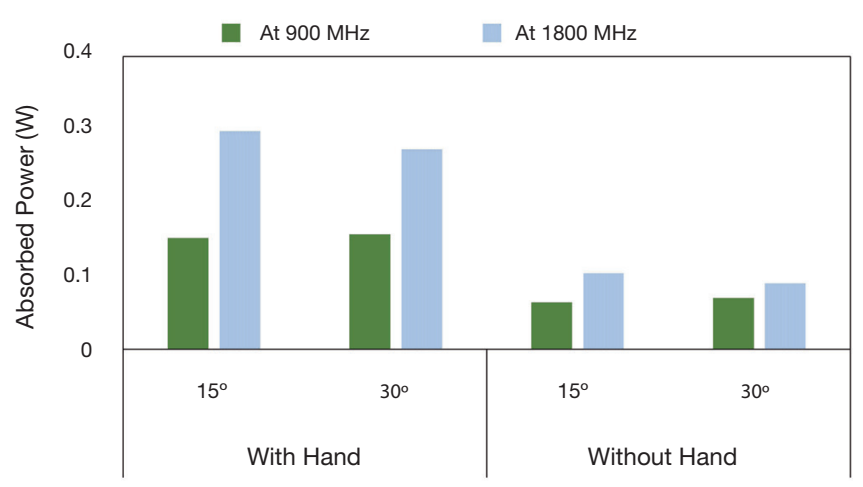

Fig. 4. Total absorbed power for tilt positions $(16 \mathrm{~mm})$.

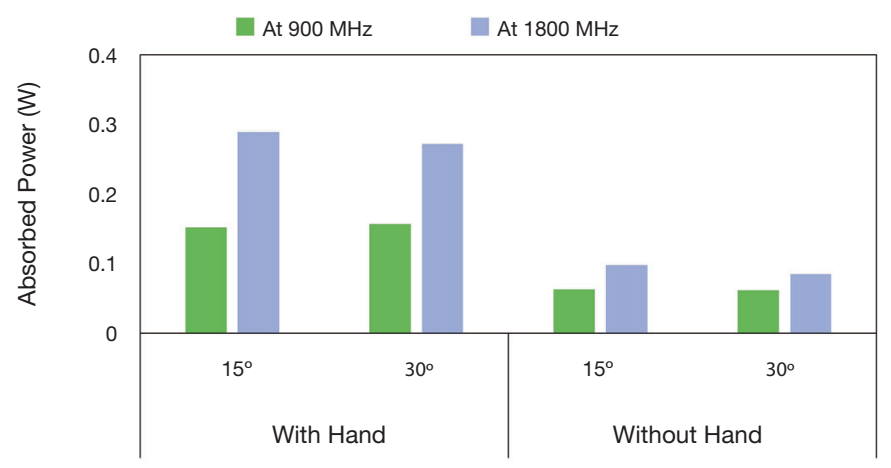

Fig. 5. Total absorbed power for tilt positions $(18 \mathrm{~mm})$.

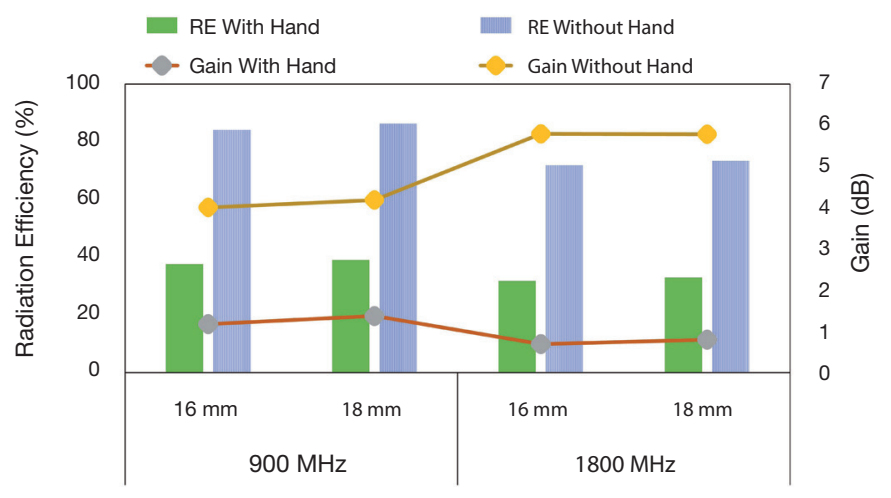

Fig. 6. Gain and radiation efficiency for cheek positions.

tions. For $15^{\circ}$ tilt position of $16 \mathrm{~mm}$, the total power absorbed by user increases $17.6 \%$ at $0.9 \mathrm{GHz}$ and $38.8 \%$ at $1.8 \mathrm{GHz}$. In addition, $30^{\circ}$ tilt position with hand of $16 \mathrm{~mm}$ contribute to increase the total absorbed power $17.4 \%$ at the lower frequency band and $36.6 \%$ at the upper frequency band. The $18 \mathrm{~mm}$ distance configuration also shows identical tendency as like as $16 \mathrm{~mm}$ distance configuration. However, the inclusion of user's hand also leads to reduce the SAR in tilt position configurations. The hand phantom absorbs a considerable amount of radiated power and hence the absorbed power in the human head is reduced.

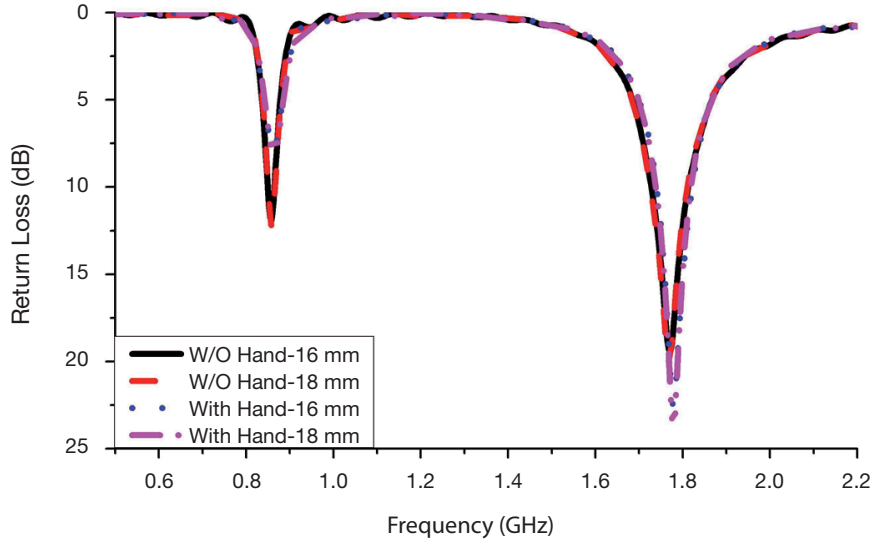

Fig. 7. Return loss characteristics for cheek positions.

\subsection{Effects of Hand on Antenna Performances}

The effects of hand on antenna performances were evaluated comprising two performance parameters: radiation efficiency and gain. Also, the results related to the antenna performance have been presented in two categories in this paper.

\subsubsection{Cheek Position Analysis}

The performance parameters of mobile phone were calculated for two different cheek positions $(16 \mathrm{~mm}$ and $18 \mathrm{~mm})$. The radiation efficiency and gain decrease due to the insertion of hand model for all configurations of cheek position. Figure 6 represents the values of radiation efficiency and gain for cheek positions. At $0.9 \mathrm{GHz}$, the radiation efficiency decreases $44.4 \%$ for $16 \mathrm{~mm}$ phone distance, and $47.3 \%$ for $18 \mathrm{~mm}$ phone distance. Additionally, the degradation of radiation efficiencies is $40.1 \%$ and $40.4 \%$ for $16 \mathrm{~mm}$ and $18 \mathrm{~mm}$ cell phone distance respectively at $1.8 \mathrm{GHz}$. Moreover, the antenna gain degrade $2.83 \mathrm{~dB}$ and $2.82 \mathrm{~dB}$ for $16 \mathrm{~mm}$ and $18 \mathrm{~mm}$ distance configuration at the lower frequency band respectively.

Sequentially, $5.1 \mathrm{~dB}$ and $4.98 \mathrm{~dB}$ antenna gain degradation occur for $16 \mathrm{~mm}$ and $18 \mathrm{~mm}$ phone distance configuration at the upper frequency band respectively. The return loss characteristics of antennas for all configurations of cheek position are plotted in Figure 7. The results indicate a little bit variation in resonance points for with hand configurations compared to without hand configurations.

\subsubsection{Tilt Position Analysis}

The results from the tilt position analysis indicate that the radiation efficiency and gain degrade owing to insertion of the hand phantom as the cheek position analysis. The radiation efficiency is reduced $41.5 \%$ and $37.4 \%$ for $15^{\circ}$ tilt configuration at $0.9 \mathrm{GHz}$ in case of $16 \mathrm{~mm}$ and $18 \mathrm{~mm}$ phone distance configuration respectively as shown in Figure 8 . Sequentially, $40.8 \%$ and $40 \%$ degradation can be found for the radiation efficiency at $1.8 \mathrm{GHz}$ for $15^{\circ}$ tilt position of $16 \mathrm{~mm}$ and $18 \mathrm{~mm}$ distance configuration respectively. For the increment in tilt angle (from $15^{\circ}$ to $30^{\circ}$ ), radiation efficiency increases little bit for both with hand and without hand configurations. Additionally, the anten- 
na gain degrades $1.6 \mathrm{~dB}$ at $0.9 \mathrm{GHz}$ and $3.95 \mathrm{~dB}$ at $1.8 \mathrm{GHz}$ for $15^{\circ}$ tilt position of $16 \mathrm{~mm}$ as indicated in Figure 9. The results also indicate that the antenna gain decreases a little bit due the increment in tilt angle $\left(15^{\circ}\right.$ to $\left.30^{\circ}\right)$. Moreover, the radiation efficiency and gain improve little bit due to increase in cell phone distance. With the variation of tilt angle, antenna performance parameters show little variation.

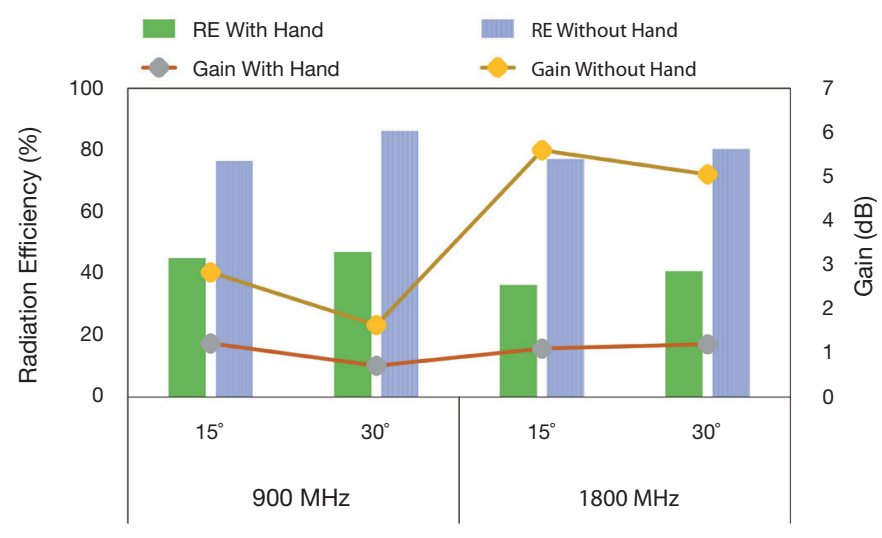

Fig. 8. Gain and radiation efficiency for tilt positions $(16 \mathrm{~mm})$.

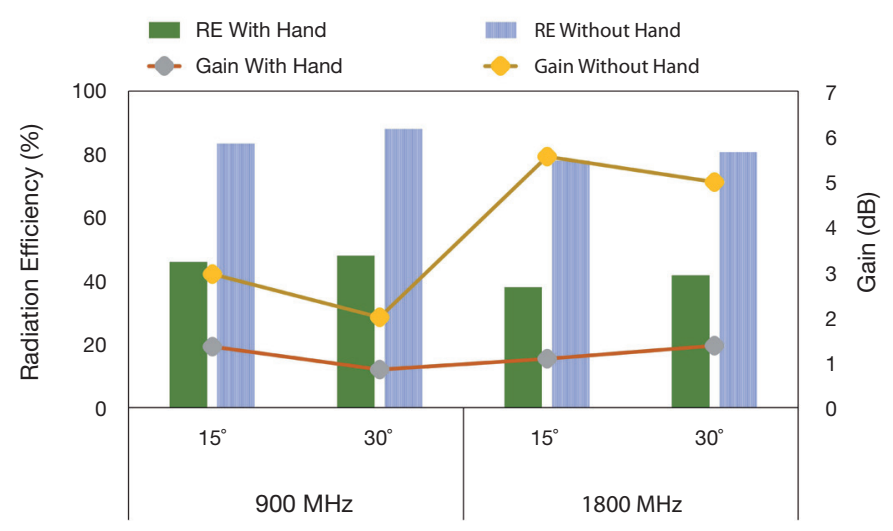

Fig. 9. Gain and radiation efficiency of tilt positions (18 $\mathrm{mm})$.

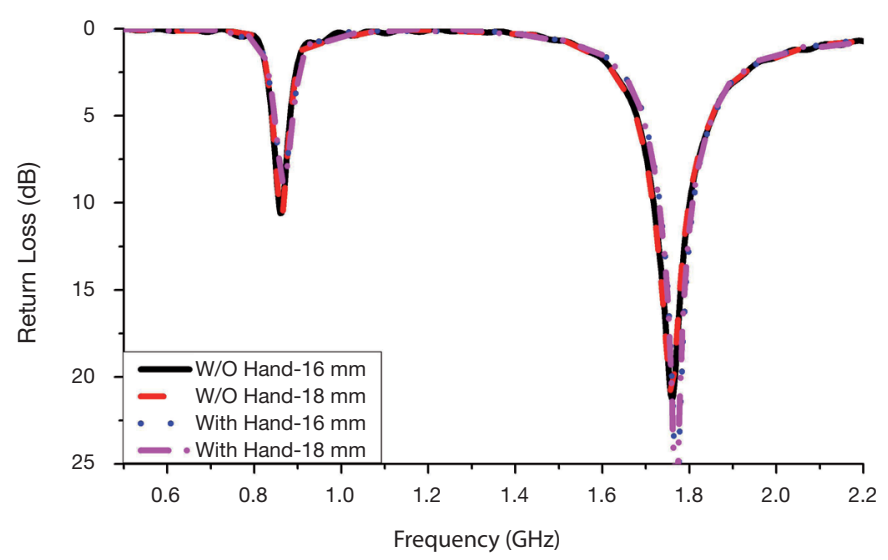

Fig. 10. Return loss curves for $15^{\circ}$ tilt positions.
The return loss characteristics of antennas for all configurations of $15^{\circ}$ tilt position are plotted in Figure 10. The results indicate a little bit variation in resonance points for with hand configurations compared to without hand configurations.

\section{Conclusions}

In this study, the effects of the human hand on the SAR values and antenna performances have been presented using mobile handset of PIFA antenna. The results of all configurations show similar tendency that the user's hand affects the SAR values greatly at the lower frequency band and slightly at the upper frequency band. The maximum $27.2 \%$ SAR reduction is observed due to the inclusion of the user's hand in both cheek and tilt position. Moreover, the hand phantom leads to increase the total power absorption more than two times in both cheek and tilt position configurations. The hand phantom also leads to degrade the antenna performances significantly. More than $40 \%$ degradation in radiation efficiency is observed both for cheek and tilt positions. In addition, gain degradation is occurring, where the gain at the upper frequency band decreases extremely in both cheek and tilt positions of talk mode.

\section{References}

Al-Mously, S.I., \& Abousetta, M.M. (2009). Anticipated impact of handhold position on the electromagnetic interaction of different antenna types/positions and a human in cellular communications. International Journal of Antennas and Propagation, 2008. DOI:10.1155/2008/102759

Commission, F.C., others. (2001). Evaluating compliance with FCC guidelines for human exposure to radiofrequency electromagnetic fields. Washington, DC: FCC. Tech. Rep. Suppl. C to OET Bulletin 65.

Faruque, M.R.I., Husni, N.A., Islam, M.T., \& Misran, N. (2014). Effects of Mobile Phone Radiation onto Human Head with Variation of Holding Cheek and Tilt Positions. Journal of Applied Research and Technology, $12,871-876$.

Hossain, M.I., Faruque, M.R.I., \& Islam, M.T. (2015). Analysis on the effect of the distances and inclination angles between human head and mobile phone on SAR. Progress in Biophysics and Molecular Biology. DOI: 10.1016/j.pbiomolbio.2015.03.008

Hossain, M.I., Mohammad, R.I.F., Islam, M.T., \& Hanafi, N.H.M. (2014). Application of auxiliary antenna elements for SAR reduction in the human head. Advanced Materials Research, 974, 288-292.

Ilvonen, J., Kivekas, O., Holopainen, J., Valkonen, R., Rasilainen, K., \& Vainikainen, P. (2011). Mobile terminal antenna performance with the user's hand: Effect of antenna dimensioning and location. Antennas and Wireless Propagation Letters, IEEE, 10, 772-775.

Kivekas, O., Ollikainen, J., Lehtiniemi, T., \& Vainikainen, P. (2004). Bandwidth, SAR, and efficiency of internal mobile phone antennas. IEEE Transactions on Electromagnetic Compatibility, 46, 71-86.

Li, C.H., Ofli, E., Chavannes, N., \& Kuster, N. (2009). Effects of hand phantom on mobile phone antenna performance. IEEE Transactions on Antennas and Propagation, 57, 2763-2770.

Okoniewski, M., \& Stuchly, M.A. (1996). A study of the handset antenna and human body interaction. IEEE Transactions on Microwave Theory and Techniques, 44, 1855-1864.

Quispe-Siccha, R.M., Reyes-Ramírez, B., García-Segundo, C., Hevia-Montiel, N., Arámbula-Cosío, F., Sato-Berrú, R., \& Flores-Flores, J.O. (2012). Implementation of Polyvynil-Alcohol Mixed With Nano-Particles as a Near Representation of Biological Tissue: Ultrasonic and Photo-Thermal Study. Journal of Applied Research and Technology, 10, 63-72. 
Repacholi, M.H. (2001). Health risks from the use of mobile phones. Toxicology Letters, 120, 323-331.

Valkonen, R., Ilvonen, J., Rasilainen, K., Holopainen, J., Icheln, C., \& Vainikainen, P. (2011). Avoiding the interaction between hand and capacitive coupling element based mobile terminal antenna. In: Proceedings of the 5th European Conference on Antennas and Propagation (EUCAP) (pp. 2781-2785). IEEE.
Vehovsky, R., Pokorny, M., \& Pitra, K. (2014). User Hand Influence on Properties of a Dual-Band PIFA Antenna. Radioengineering, 23, 819823.

Zhao, K., Zhang, S., Ying, Z., Bolin, T., \& He, S. (2013). Reduce the handeffect body loss for LTE mobile antenna in CTIA talking and data modes. Progress In Electromagnetics Research, 137, 73-85. 